\title{
XXVII. The theory of magnetism and the absurdity of diamagnetic polarity
}

\section{J. Parker M.A.}

To cite this article: J. Parker M.A. (1891) XXVII. The theory of magnetism and the absurdity of diamagnetic polarity, Philosophical Magazine Series 5, 32:195, 192-203, DOI: 10.1080/14786449108621411

To link to this article: http://dx.doi.org/10.1080/14786449108621411

曲 Published online: 08 May 2009.

Submit your article to this journal $[\pi$

Џll Article views: 3

Q View related articles $\square$ 
of the constancy of $\mu$ during a cycle be denied me, and if I must assume the possibility of its being wrong, still $\frac{L L^{\prime}-M^{2}}{L^{\prime}}$ must be more nearly constant than $\mu$; for it is equal to $2 \mathrm{~L} y$. And if $\mu$ increases and therefore L increases, $y$ will certainly diminish, and if $\mathrm{L}$ diminishes $y$ will certainly increase.

XXVII. The Theory of Magnetism and the Absurdity of Diamagnetic Polarity. By J. PARKer, M.A., Fellow of St. John's College, Cambridge* .

THE most unsatisfinctory part of the theory of magnetism 1 is that which refers to the so-called diamagnetic bodies. This part of the theory is so beset with absurdities and contradictions that it is necessary to examine it closely and to point out the true explanation of the behaviour of the so-called diamagnetic bodies before we can give the general theory.

I published a short and somewhat premature paper on diamagnetism in the Philosophical Magazine for May 1889. A few weeks afterwards my ideas on the subject had ripened into their present form; but I determined to publish no more about it until after the appearance of my book on 'Elementary Thermodynamics,' which I was then intending to write. Finding, however, that my book was not likely to be finished as soon as I had expected, I published a second short paper on diamagnetism in the Philosophical Magazine for July 1890 , which I thought would be sufficient to explain my ideas until I could give the subject the attention it deserved.

My second paper on diamagnetism was criticised, as I have since found, by Dr. Lodge with a great display of rhetoric in the next number of the Philosophical Magazine; but I was then so occupied that I did not see or hear of the criticism until the following November. When I then came to read it, I did not find anything which required me to modify any of my ideas on the subject in the slightest degree, and $\mathbf{I}$ concluded that Dr. Lodge had misunderstood my paper.

It is now proposed to consider the subject carefully and completely, by which means, it is hoped, the disputed points will be settled to the satisfaction of everybody. In so doing, I shall endeavour to prevent all misconception by making, my arguments as clear and simple as possible. For this reason I shall employ none but the simplest mathematics,

\footnotetext{
* Communicated by the Author.
} 
and shall avoid unnecessary references to the physical theories of magnetism.

To begin, the property which distinguishes the so-called diamagnetic bodies (like bismuth and copper) from the other, or paramagnetic bodies (like iron), is shown by the following classical experiment. A small bar, $A$, of the substance to be experimented on, is hung by a thread between the poles, NS, of a powerful electromagnet and then the magnet is excited. If the bar $\mathrm{A}$ be one of the so-called diamagnetic substances, it will set its length at right angles to the line joining the two poles of the electromagnet, as shown in the second accompanyFig. 1. ing figure, which is supposed to be a view as seen by an eye looking from above. If, however, the bar A be one of the paramagnetic substances, it sets its length along the line joining the two poles of the electromagnet,

Instead of taking the above fundamental experiment as our starting-point, I prefer, for the sake of simplicity, to take another Fig. 2. which is easier to understand. The second

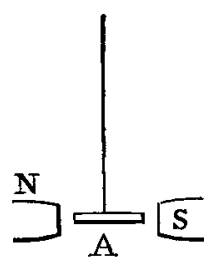
experiment has not yet been performed ; but it is admissible to make it the basis of our discussion, for not only is it equivalent to the classical experiment described above, but the inferences drawn from both will be the same.

In our ideal experiment, we take a powerful electromagnet whose poles are at a considerable distance apart, and hang a small piece, $M$, of the substance to be tested by a thread near either pole, $\mathrm{P}$, of the electromagnet. Then on exciting the magnet, it is found that if $\mathrm{M}$ be a piece of iron, it is drawn nearer the pole $P$, while if it be a piece of bismuth, it would be driven further away from it.

Fig. 3.

The so-called explanation which is usually given of these phenomena may be divided into two parts :-

(a) It is supposed (that is, assumed) that the pressure of the air is strictly uniform all over the surface of $M$, from which it would follow that the pressure of the air can have no influence in causing the body $\mathrm{M}$ to approach or recede from the excited pole $P$.

(b) It is then supposed, as a necessary consequence, that when the electromagnet is excited, a piece of iron is attracted to the pole $\mathrm{P}$, and that a piece of bismuth is repelled.

Phil. Mag. S. 5. Vol. 32. No. 195. August 1891. 


\section{Mr. J. Parker on the Theory of Magnetism and}

Experiments, which need not here be described, have been made with many solids and with liquids and gases, from which it is inferred that all substances are susceptible to magnetic influence, and it is supposed necessary to divide them into two classes: paramagnetic, those which, when placed near one pole of a magnet, are supposed to be attracted; diamagnetic, those which, under the same circumstances, are supposed to be repelled.

I first of all deny that (a) can be correct. For let an ideal right circular cylinder $\mathrm{PQRS}$, indefinitely small in all its dimensions, be described in the air anywhere near the pole $\mathrm{P}$ of the electromagnet such that at no point within the cylinder is the magnetic force at right angles to the axis of the cylinder. Also let the ends $\mathrm{PQ}, \mathrm{RS}$ of the cylinder be normal sections and suppose that the radius of each is indefinitely small in comparison with the length of the cylinder. Then since, in the state of equilibrium, the pressure of the magnetized air on any surface is evidently Fig. 4. normal to that surface, just as if gravitation

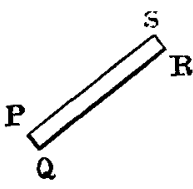
were the only action at a distance, it follows that the pressure of the air, per unit of area, at the end $\mathrm{PQ}$ of the cylinder is not the same as the pressure at the end RS. Thus in the space around the excited pole $\mathbf{P}$, the pressure of the magnetized air varies from point to point, and therefore the assumption (a) cannot be correct.

The explanations $(a)$ and $(b)$ must therefore be modified as follows :- It must be admitted that in the above experiment the pressure of the magnetized air is not quite uniform over the surface of $M$, but it may be supposed that the resultant pressure is so small in comparison with the action of the pole $\mathrm{P}$ that it is still necessary to divide bodies into two classes, viz. those which are attracted by the pole $P$, and those which are repelled by it.

Now if we recollect that the so-called diamagnetic bodies (like bismuth), which, when the magnet is excited, recede from the pole $\mathrm{P}$, are caused to do so by a force which is evidently very minute, it will be easy to show that a very slight inequality (too small to be detected) in the pressure of the air on the surface of $M$ is quite competent to produce the phenomena observed. Diamagnetic bodies may therefore be really attracted to the pole $\mathrm{P}$, and only caused to recede from it by an excess in the pressure of the air on the side of $\mathbf{M}$ next to $\mathrm{P}$ over the pressure on the side remote from $\mathrm{P}$; just as a balloon is attracted to the earth and only caused to ascend by the pressure of the air. 
To make our argument simple, let us suppose that in our ideal experiment the body $\mathrm{M}$ is a cube each of whose edges is one centimetre, and let it be hung so symmetrically with respect to the magnet pole $\mathrm{P}$ that it is only on the face $\mathrm{Y}$, next to $P$, and on the opposite face $Z$, that the pressure of the air need be considered. Also, for the sake of simplicity, let us suppose the pressure to be uniform over each of these faces. Then if the pressure of Fig. 5. the air on the face $Y$ exceed that on the face $Z$ by the 1000th part of an atmo, the resultant pressure tending to drive $M$ away from $P$ will be rather over 1 gramme weight. If $M$ be of bismuth, the most strongly pronounced of the so-called diamagnetic substances, the mass of the cube will be nearly 10 grammes. The resultant pressure of the air is therefore about $\frac{1}{10}$ th of the weight of the cube. It will, therefore, be capable, in one second, of generating in the cube a velocity of 98 centimetres per second, and of causing it to move from rest through 49 centimetres. In $\frac{1}{4}$ of a second, it would generate in the cube a velocity of $24 \cdot 5$ centimetres $\left(9 \cdot i^{\circ}\right.$ inches $)$ per second, and cause it to move through 3 centimetres ( 1.2 inches). If the pressure on the face $\mathrm{Y}$ exceed that on the face $\mathrm{Z}$ by the 10,000 th of an atmo, the resultant pressure tending to drive the cube away from $P$ will be about the $\frac{1}{100}$ th part of its weight. This would be sufficient, in one second, to give the cube a velocity of $9 \cdot 8$ centimetres (3.8 inches) per second, and to cause it to move through 4.9 centimetres. In $\frac{1}{2}$ second it would give the cube a velocity of 4.9 centimetres ( 1.9 inches) per second, and cause it to move through $1 \cdot 2$ centimetres $(\cdot 46$ inch).

The preceding calculations may well throw doubt on the common theory of diamagnetism, but the theory has still one apology left. It is found that diamagnetic bodies retain their characteristic property in a comparative "vacuum" of 2 or 3 millimetres of mercury (say the ${ }_{300}$ th of an atmo). To explain this, we have only to observe that if, in our ideal experiment, the attraction of the pole $\mathrm{P}$ on the body $\mathrm{M}$ were strictly zero, the body $M$ would be driven away from the excited pole $\mathrm{P}$ by the pressure of the air however good the "vacuum" might be, short of absolute perfection. Hence if the attraction of $P$ on $M$ be not strictly zero but exceedingly small, it will be necessary to reduce the density of the air very nearly to zero before this attraction can make itself manifest.

Having now shown that the common notion of diamagneO 2 
tism rests on insufficient experimental evidence, or rather, on none at all, I may add that no one has ever attempted to support the notion by theory. This will prepare the way for my alternative idea, based on theory, that every substance placed near one pole of a magnet is attracted by the pole when the magnet is excited and can only be prevented from approaching the pole by some antagonistic cause, such as the pressures exerted by the contiguous objects. In the common theory of diamagnetism, it is supposed that bismuth is more powerfully repelled by a magnet pole than any other substance; according to my theory, bismuth is more feebly attracted than any other substance.

Before giving the theoretical grounds of the new theory, I cannot help observing that those who think the old theory of diamagnetism sufficient, should try to prove that the ascent of a balloon in the air is due to the diagravitational repulsion of the earth. Those who accept the new theory, will be content to regard diagravitation and diamagnetism as companion absurdities.

I was first led to reason theoretically on diamagnetism by reading the following remarkable article in Prof. Tait's 'Sketeh of Thermodynamies':- " The commonly received opinion, that a diamagnetic body in a field of magnetic force takes the opposite polarity to that produced in a paramagnetic body similarly circumstanced, is thus attacked by Thomson by an application of the principle of energy. Since all paramagnetic bodies require time for the full development of their magnetism, and do not instantly lose it when the magnetizing force is removed, we may, of course, suppose the same to be true for diamagnetic bodies; and it is easy to see that in such a case a homogeneous non-crystalline diamagnetic sphere rotating in a field of magnetic force would, if it always tended to take the opposite distribution of magnetism to that acquired by jron under the same circumstances, be acted upon by a couple constantly tending to turn it in the same direction round its centre, and would therefore be a source of the perpetual motion."

This argument, which, for some unaccountable reason, seems to have been entirely neglected, requires to be slightly modified; for no notice is taken of the frictional resistance of the air to the rotation of the sphere, and it is not shown that the motion supposed to be thus derived in unlimited quantities may not be due to the absorption of heat at one temperature and its partial rejection at a lower. These defects may be obviated by supposing the sphere placed in a "perfect vacuum," and making provision to prevent the system 
absorbing or giving out heat except at some constant absolute temperature $\theta$. Also, for the sake of simplicity, we may suppose the sphere connected with mechanism, on which it does just so much work that its angular velocity of rotation remains constant. Under these conditions the system will assume an inviriable state. If we then suppose that $\mathrm{W}$ is the mechanical work done on the system in any time, and $Q$. the amount of heat absorbed, the principle of energy will give, since there is no change of state,

$$
\mathrm{W}+\mathrm{Q}=0 \text {. }
$$

Now since work is obtained from the system, $W$ is negative.

We see, therefore, that $Q$ is positive.

But by Carnot's principle, we have

$$
\frac{\mathrm{Q}}{\theta}<0, \text { or } \mathrm{Q}<0 \text {. }
$$

Thus Thomson's ideal experiment is inconsistent with both the principle of energy and with Carnot's principle, and the hypothesis of diamagnetic polarity is therefore disproved.

The argument adopted in the two papers I have published on diamagnetism in the Philosophical Magazine is only a modified form of Thomson's, but possesses the advantage of simplicity. It may be described thus : Take a piece of bismuth (or of any of the so-called diamagnetic substances) and place it within the action of a strong steel magnet whose poles are so far apart that only one of the poles, $\mathrm{P}$ say, exerts any sensible force on the bismuth. Then let the steel magnet be made immovable, and suppose the piece of bismuth held by a pair of pincers, or in some other way, so that it can either be kept at rest or moved about at will. Next, let the air be exhausted in the space about the bismuth until the "vacuum" is perfect, and, lastly, let means be taken to prevent the absorption or rejection of heat, except at some constant temperature $\theta$.

If the piece of bismuth is held for some time in any position $X$ and then suddenly moved to a new position $Y$, nearer to $P$, the magnetization of the bismuth will, of course, become ultimately stronger in the position $\mathrm{Y}$ than it was in the more remote position $\mathrm{X}$; but, since magnetization requires time for its development, the change of position may be effected so rapidly that the magnetization of the bismuth has scarcely time to alter before the new position $\mathrm{Y}$ is reached. We thus see that the amount of mechanical work which can be obtained from the system of the steel magnet and the piece of bismuth during a given change of position depends, 
among other things, on the speed at which the change of position is made. Suppose then, if possible, that the bismuth is repelled by the pole $\mathrm{P}$, and let the system be made to undergo the following cycle of operations in two different ways :-

(1) When the piece of bismuth is in equilibrium in any position $\mathrm{X}$ at the uniform temperature $\theta$, let it be moved to some other position $\mathrm{Y}$, nearer $\mathrm{P}$, so slowly that at every point of the path the bismuth is magnetized to the full extent and the temperature constant. The work done on the system in this operation will be positive, and may be written $W$.

Then let the piece of bismuth return slowly from $\mathrm{Y}$ to $\mathrm{X}$ by the previous path reversed. The whole of the work $W$ will thus be returned by the system, so that, on the whole, there is neither loss nor gain of mechanical work during the cycle.

(2) Let the piece of bismuth be moved from $\mathrm{X}$ to $\mathrm{Y}$ so rapidly that the magnetization has scarcely time to change before the ehange of position is complete. The work that must be expended for this purpose is obviously $<W$; in fact, if the position $X$ be far enough away from $P$, the work done on the system during the change of position may be practically zero. Next, let the piece of bismuth remain at rest in its new position until its magnetization has attained its permanent value. Then let it be brought rapidly back from $Y$ to $\mathrm{X}$ by the first path reversed; and, lastly, let it remain in the position $\mathrm{X}$ until the original state is attained. The mechanical work returned by the system in this way will be $>W$. Thus, on the whole, there is a gain of mechanical work during the cycle in which heat is absorbed and evolved at a constant tenperature. This being impossible, by the principle of energy and Carnot's principle, we conclude that the assumption that the piece of bismuth is repelled by the pole of the magnet is absurd.

The foregoing cycle was described in my first paper on diamagnetism; but the inference then drawn was, not that diamagnetism is impossible, but that Carnot's principle does not hold for diamagnetic bodies. In my second paper, I denied the existence of diamagnetism; but long before that time Duhem had arrived at the same conclusion, from reading my first paper, and had written a book on the subject.

I must now point out that Dr. Lodge has objected, with justice, to the preceding reasoning, that it contains a tacit assumption. Thus, even if we admit, as we are bound, that the magnetization of every body takes time to grow and to die away, it is not proved but that, in the second way of per- 
forming the experiment described above, the assumed repulsive force between the steel and the bismuth may be so affected by the rapidity of the motion that the work required to force the bismuth from the position $\mathrm{X}$ to the position $\mathrm{Y}$ may be $>W$, and not less, as we supposed.

Dr. Lodge's objection may be illustrated very simply as follows :- Let a quantity of gas be contained in a cylinder fitted with a smooth air-tight piston, and suppose it to be provided that the cylinder can absorb or give out heat freely, but only at some constant temperature $\theta$. When the whole is in equilibrium at the uniform temperature $\theta$, let the piston he pushed in so slowly that the system is constantly in equilibrium, and the temperature always uniform and equal to $\theta$. The work required for this purpose may be called W. Then let the piston be drawn out slowly until the original state is attained. The work restored will be $W$, and, on the whole, there will be neither loss nor gain of work.

Next, let the piston be pushed in as much as before, but far more rapidly. The work expended on the system in doing this will be $>W$. Now let the piston remain in its new position until the temperature has everywhere sunk to $\theta$. Then let the piston be rapidly drawn out to its original place, and then wait until the original state is brought about. The work restored by the system will be $<W$, so that there will have been a loss of mechanical work in the cycle.

Dr. Lodge's objection may be obviated by means of two assumptions :-

(1) We assume that the magnetization of a body at rest may be in a state of unstable equilibrium. For example, in the experiment with the piece of bismuth, we assume that when the bismuth is at rest in any position $\mathrm{X}$, the repulsive force of the steel magnet may have a value $f$ which does not properly belong to the position $X$, and that this force may continue unchanged so long as the bismuth is held steadily enough in the position $X$. We further assume that if the bismuth be moved slowly from the position $\mathrm{X}$, and then slowly brought back again, the repulsive force of the steel magnet will again be $f^{\prime}$, if sufficient care bas been taken to move the bismuth about steadily. When the magnetization of a body at rest is in a state of unstable equilibrium, a slight shake, by which no perceptible amount of mechanical work is done, will be sufficient to cause the magnetization to rise or sink to its proper value.

(2) We also assume that if two magnetized bodies be moved about with any velocities, the attraction or repulsion between them when they are passing through any given 
position may, if the experiment is made steadily enough, be exactly the same as if both bodies were at rest in that position with their magnetizations invariable.

In applying these assumptions to the second method of making the experiment with the piece of bismuth, let us suppose, merely for the sake of simplicity, that the magnetization of the steel is "rigid," that is, not merely unstably invariable, but invariable under all circumstances. Then let the cycle be performed as follows:-

(a) Let the bismuth be moved from $\mathrm{X}$ to $\mathrm{Y}$ so steadily that its magnetization is unaltered. It is evident that no thermal phenomenon will attend this operation, and that the work done on the system will be $<W$.

(b) The magnetization of the piece of bismuth now being in unstable equilibrium, let a slight shake, by which no perceptible amount of mechanical work is done, be given to the bismuth, in consequence of which the strength of the magnetization will be affected, and a thermal phenomenon take place. Then let the bismuth be held in the position $\mathrm{Y}$ until the temperature is again $\theta$, and the magnetization at its proper value.

(c) Next let the piece of bismuth be brought back from $\mathrm{Y}$ to $\mathrm{X}$ by the first path reversed, and so steadily that the magnetization does not change. The work restored by the system during this operation is $>\mathrm{W}$.

(d) Lastly, let a slight shake be given and hold the piece of bismuth in the position $\mathrm{X}$ until the system is in the original state.

We see therefore that if the two assumptions are made, the hypothesis of diamagnetism enables us to obtain work from a cycle in which heat can only be absorbed or given out at a given constant temperature $\theta$. We now contend that the two assumptions are admissible. We shall establish the truth of this assertion indirectly by showing what results the two assumptions lead to when applied to several of the most important propositions in magnetism and electricity.

I. If in the modified Thomson's argument, we suppose the piece of bismuth replaced by any substance $S$ which is attracted to the pole $P$, it will easily be seen that if the magnetization of $S$ increases as the distance from $P$ decreases, our two assumptions do not lead to a result in contradiction to the principle of energy and to Carnot's principle. We therefore conclude that a substance may be attracted by a magnet pole-a result which, of course, is in accordance with experiment.

It is necessary to notice another method of making the 
experiment. If the substance $\mathrm{S}$ be brought from $\mathrm{X}$ to $\mathrm{Y}$, a positive quantity of work, $W_{1}$ say, will be obtained. Then if $\mathrm{S}$ be turned round without altering the magnetization, the force between $P$ and $S$ will become repulsive, and therefore if $S$ be brought back from $Y$ to $X$ without any change in the magnetization, another positive quantity of work, $\mathrm{W}_{2}$ say, will be obtained. In this way, it might be thought, we could prove that $S$ could not be attracted by $P$, but we must recollect that work will have been expended in turning $S$ round. In fact, we may be sure that the work expended in turning $\mathbf{S}$ round, first in the position $\mathrm{Y}$, and then in the position $\mathrm{X}$, will be $>\mathrm{W}_{1}+\mathrm{W}_{2}$.

II. Generally, if the substance M, when removed to a sufficient distance from the pole $\mathrm{P}$, is in the neutral state, it can easily be proved that when $M$ is near $P$, and its magnetization in stable equilibrium, $\mathrm{M}$ must be attracted by $\mathrm{P}$ with a force which increases as the distance from $P$ decreases. Such a substance is defined to be "perfectly soft," and since unstable states of magnetization do not occur in practice, we may say that a "perfectly soft" substance can only be attracted by a magnet pole.

Of course, a substance $S$ may be repelled by the pole $\mathrm{P}$, but in that case, it can easily be shown, the magnetization of $S$ could not be zero when it is at a great distance from $P$. The substance $\mathrm{S}$ would then be a permanent magnet, or its magnetization would consist of a permanent part combined with a temporary part induced by the influencing pole $P$.

III. If we now suppose the pole $P$ replaced by a positively electrified body and the piece of bismuth by a negatively electrified body B, the force between the two bodies will be attractive, and when $B$ is moved from $X$ to $Y$ in such a way that the distributions of the electrifications are in stable equilibrium, the attraction on $B$ in the position $\mathrm{Y}$ will be greater than if the distributions had not been changed. Hence if $W$ be the positive quantity of work yielded by the system when $\mathrm{B}$ is moved from $\mathrm{X}$ to $\mathrm{Y}$ in such a way that at every instant the distributions are in stable equilibrium, then when $B$ is moved from its initial position $X$, in which the distributions are stable, to the second position $Y$, without any change in the distributions, the work yielded by the system will be $<W$. Also if, after causing the distributions in the position $\mathrm{Y}$ to become stable, we bring $\mathrm{B}$ back from $\mathrm{X}$ by the previous path reversed without any change in the distributions, the work so done on the system will be $>W$. Consequently, if the system is now caused to resume its original state, a complete cycle will have been undergone, and a posi- 
tive quantity of work expended in it, which is in accordance with the principles of thermodynamies.

IV. Next, let both bodies be positively electrified, and suppose that they are at such a distance from one another throughout the experiment that the force between them is always repulsive. Then, when $B$ is moved from $\mathrm{X}$ to $\mathrm{Y}$ in such a way that the distributions are constantly in stable equilibrium, the repulsion on $\mathrm{B}$ in the position $\mathrm{Y}$ will be less than if the distributions had not changed. (In the case of a supposed diamagnetic "soft substance," it will be recollected that the repulsive force in the position $\mathrm{Y}$ was greater than if there had been no change in the magnetization.) From this it is easily seen that electric repulsion is in accordance with the principles of thermodynamics.

V. Again, if we assume the principle of the conservation of electricity, which can be partially obtained as a theoretical deduction from the principle of energy and Carnot's principle, our two assumptions enable us to find expressions for the energy and entropy of an electrified system at rest in which the distribution of electricity is in equilibrium, stable or unstable. Then we can easily find the condition of electric stability on an insulated homogeneous conductor. For if $\mathrm{P}, \mathrm{Q}$ be two points of such a conductor, our expressions show that if the potential have a higher value at $P$ than at $Q$, it will be possible for electricity to pass, of itself, from $P$ to $Q$, but not from $\mathrm{Q}$ to $\mathrm{P}$. Conversely, if the potential at $\mathrm{P}$ be less than at $Q$, electricity will be able to pass, of itself, from $Q$ to $P$, but not from $P$ to $Q$. Suppose, for example, that the potential at $\mathrm{P}$ is greater than at $\mathrm{Q}$ : then a slight shake may cause a passage of electricity from $P$ to $Q$, and this will diminish the potential at $P$ and increase it at $Q$. Thus the potential tends to become uniform throughout the conductor, and when it has become uniform, the distribution is stable.

VI. Again, our expressions for the energy and entropy enable us to discuss the conditions of stability of two conductors A, $B$, separately homogeneous, which are in contact with each other, and isolated from all other bodies. For if $\mathrm{P}$ be a point in the body $A$, and $Q$ a point in the body $B$, our expressions show that if the potential at $\mathrm{P}$ exceed the potential at $\mathrm{Q}$ by more than a certain amount, electricity will be able, of itself, to pass from $\mathrm{P}$ to $\mathrm{Q}$, but not from $\mathrm{Q}$ to $\mathrm{P}$; and conversely. Thus the ccndition of stability is that the potential should be uniform throughout each conductor, and that the two values should differ according to a certain law; all which is verified by experiment. Again, if the distributions be indefinitely. near stable, and we suppose a small charge to pass from one conductor to the other, our theory indicates 
that it will be necessary to impart or take away heat to keep the temperature of the junction of the two bodies constant. In other words, the theory gives both the difference of potential and the Peltier effect.

Now it is important to remark that by merely assuming our expressions for the energy and entropy to hold for stable distributions and without making any further use of the conception of unstable distributions, we can easily obtain the very same expressions for the difference of potential and the Peltier effect as before.

In like manner, we have two methods of discussing the question of a difference of potential and the law of the Thomson effect, in the case of two unequally heated portions of the same substance in contact, and both methods give the same results.

VII. Lastly, we have two ways of investigating Helmholtz's theory of the galvanic battery, and both lead to the same conclusions. This theory, it is true, has been questioned; but the objections appear to rest on misconceptions; and, besides, the theory does not seem to have been yet sufficiently explained. However, I do not propose to consider the subject any further at present, but merely wish to point out that we have two ways of getting the same result.

I have now, I consider, conclusively shown that my two assumptions are legitimate; and yet, at the same time, I acknowledge the importance of Dr. Lodge's objection, in requiring us to examine the tacit assumptions which lie at the root of all thermodynamical investigations of electricity and magnetism.

To conclude our discussion on diamagnetism, I observe that, so far as I am aware, no one has ever attempted to advance any serious theoretical or other arguments in favour of the common notion of diamagnetism. The theory appears to have owed its origin to the want of a theoretical examination of the subject, and to have been maintained from an excusable veneration for an old theory and a belief in the infallibility of the older scientists. Yet it has been occasionally questioned from the first. Duhem, in his book on diamaguetic bodies, mentions Becquerel and Plücker as haring objected to the theory. Even in England there are persons who have never accepted it, and Dr. Lodge limself seems to be aware that it will not stand examination; for in his criticism on $\mathrm{my}$ two previous papers on the subject, he appears to rely cbiefly on the strength of his rhetoric, and on an appeal to the name of Faraday.

I now proceed to the next part of the paper.

[To be continued.] 